\title{
High throughput microwell spectrophotometric assay for olmesartan medoxomil in tablets based on its charge-transfer reaction with DDQ
}

\author{
IBRAHIM A. DARWISH ${ }^{1 *}$ \\ TANVEER A. WANI ${ }^{1}$ \\ NASR Y. KHALIL ${ }^{1}$ \\ HAMDY M. ABDEL-RAHMAN ${ }^{2}$ \\ ${ }^{1}$ Department of Pharmaceutical Chemistry \\ College of Pharmacy, King Saud University \\ P.O. Box 2457, Riyadh 11451, Saudi Arabia \\ 2 Department of Medicinal Chemistry \\ Faculty of Pharmacy, Assiut University \\ Assiut 71526, Egypt
}

Accepted November 2, 2013
The study describes the development and validation of a new microwell-based spectrophotometric assay for determination of olmesartan medoxomil (OLM) in tablets. The formation of a colored charge-transfer (CT) complex between OLM as an n-electron donor and 2,3-dichloro-5,6-dicyano-1,4-benzoquinone (DDQ) as a $\pi$-electron acceptor was investigated, and employed as the basis for the development of the new assay. The proposed assay was conducted in 96-microwell plates. The absorbance of the colored-CT complex was measured at $460 \mathrm{~nm}$ with a microplate reader. Optimum conditions of the reaction and the analytical procedures of the assay were established. Under the optimum conditions, a linear relationship with a good correlation coefficient was found between the absorbance and the concentration of OLM in the range of 2-200 $\mu \mathrm{g}$ per well. The limits of detection and quantitation were 0.53 and $1.61 \mu \mathrm{g}$ per well, respectively. No interference was observed from the excipients present in OLM tablets or from hydrochlorothiazide and amlodipine besylate that were co-formulated with OLM in some of its formulations. The assay was successfully applied to the analysis of OLM in tablets with good accuracy and precision. The assay described herein has a great practical value in the routine analysis of OLM in quality control laboratories, since it has a high throughput property and consumes low volumes of organic solvent. It thus offers a reduction in the exposure of analysts to the toxic effects of organic solvents, as well as a reduction in the cost of analysis.

Keywords: olmesartan medoxomil, spectrophotometry, charge-transfer complex, 2,3-dichloro-5,6-dicyano-1,4-benzoquinone, microwell assay

\footnotetext{
* Correspondence; e-mail: idarwish@ksu.edu.sa
} 


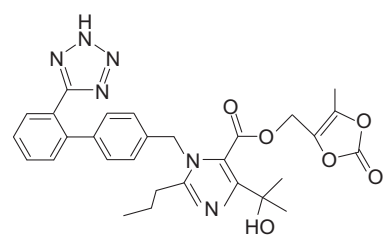

Olmesartan medoxomil (OLM)

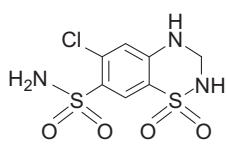

Hydrochlorothiazide

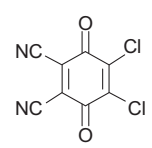

2,3-Dichloro-5,6-dicyano-1,4benzoquinone (DDQ)

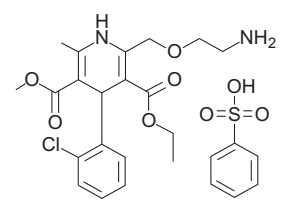

Amlodipine besylate

Fig. 1. Chemical structures of olmesartan medoxomil (OLM), 2,3-dichloro-5,6-dicyano-1,4-benzoquinone (DDQ) reagent and the co-formulated drugs (hydrochlorothiazide and amlodipine besylate).

Olmesartan medoxomil [OLM, (5-methyl-2-oxo-2H-1,3-dioxol-4-yl)methyl 4-(2-hydroxy-propan-2-yl)-2-propyl-1-(\{4-[2-(2H-1,2,3,4-tetrazol-5-yl)phenyl]phenyl $\}$ methyl)$1 \mathrm{H}$-imidazole-5-carboxylate, Fig. 1], is the newest member of non-peptide angiotensin II receptor antagonists used worldwide in the treatment of hypertension. It is an ester prodrug, which is completely and rapidly hydrolyzed to the active acid form, olmesartan. OLM exerts its action mainly via a selective blockade action on AT1 receptors and the consequent reduced pressor effect of angiotensin II $(1,2)$. OLM may be used alone or in combination with other antihypertensive agents (e.g., hydrochlorothiazide).

OLM has not yet been officially described in any pharmacopoeia. A literature survey revealed that several analytical methods were reported for its determination. These methods include high-performance thin-layer chromatography (3), liquid chromatography (3-9), and capillary zone electrophoresis (10). Also, a few spectrophotometric methods have been reported for the analysis of OLM in pharmaceutical tablets (11-13). Unfortunately, these methods suffer from major drawbacks such as decreased selectivity due to measuring the native light absorption of OLM in the blue-shifted ultraviolet region, which might be subjected to interferences $(11,12)$. Besides, the tedious liquid-liquid extraction procedures use large volumes of organic solvents in the methods based on the formation of ion-pair associates (13). Therefore, the development of a new alternative spectrophotometric method for the determination of OLM in pharmaceutical formulations is essential.

The charge-transfer (CT) reaction between the electron-donating pharmaceuticals and electron-accepting reagents is widely employed as the basis for the development of visible spectrophotometric methods (14-16). These facts promoted our interest in employing the CT-reaction as a basis for the development of a new spectrophotometric method for OLM determination. However, the CT-based spectrophotometric methods 
employing the conventional spectrophotometers are not automated and consequently their throughput is low; thus their applications in pharmaceutical quality control laboratories are limited. Moreover, these methods suffer from the consumption of large volumes of organic solvents, which leads to high analysis costs, and more importantly, the exposure of analysts to the toxic effects of organic solvents $(17,18)$.

The present study describes the investigation of the CT reaction of OLM, as an electron donor, with the $\pi$-electron acceptor 2,3-dichloro-5,6-dicyano-1,4-benzoquinone (DDQ) to be used in the development of a new 96-microwell spectrophotometric assay for the determination of OLM in tablets.

\section{EXPERIMENTAL}

\section{Apparatus}

A microwell-plate absorbance reader (ELx 808, Bio-Tek Instruments Inc., USA) was used for all measurements in 96-microwell plates. A UV-1601 PC (Shimadzu, Japan) ultraviolet-visible spectrophotometer with matched $1 \mathrm{~cm}$ quartz cells was used for recording the absorption spectra. 96-Microwell plates were a product of Corning/Costar Inc. (USA). A Finnpipette adjustable 8-channel-pipette was obtained from Sigma Chemical Co. (USA).

\section{Chemicals and tablets}

OLM was obtained from AK Scientific Inc. (USA). Amlodipine besylate and hydrochlorothiazide were obtained from Sigma Chemical Co. (USA). DDQ (Merck, Germany) was $8.8 \times 10^{-2} \mathrm{~mol} \mathrm{~L}^{-1}$ in methanol; it was prepared fresh daily. Olmetec ${ }^{\circledR}$ tablets (SAJA Pharmaceuticals, Saudi Arabia) labeled to contain $20 \mathrm{mg}$ of OLM were obtained from the local market. Azor ${ }^{\circledR}$ tablets labeled to contain $20 \mathrm{mg}$ of OLM and $5 \mathrm{mg}$ of amlodipine besylate and Tribenzor $^{\circledR}$ tablets labeled to contain 20, 5, and $12.5 \mathrm{mg}$ of OLM, amlodipine besylate and hydrochlorothiazide were products of Daiichi Sankyo Europe $\mathrm{GmbH}$, Germany and both obtained from the local market.

\section{Preparation of standard and sample solutions}

Stock standard solutions. Ten mg of OLM was accurately weighed into a 5-mL calibrated flask, dissolved in $2 \mathrm{~mL}$ methanol and completed to volume with the same solvent. This stock solution was diluted with methanol to obtain suitable concentrations lying in the linear range of the assay. The OLM solutions were found to be stable for at least two weeks when kept in a refrigerator.

Tablet solutions. - Twenty tablets were weighed and finely powdered. A quantity of powder equivalent to $20 \mathrm{mg}$ of OLM was transferred into a $10-\mathrm{mL}$ calibrated flask, dissolved in $4 \mathrm{~mL}$ methanol, swirled and sonicated for $5 \mathrm{~min}$, completed to volume with methanol, shaken well for $15 \mathrm{~min}$, and filtered. The first portion of the filtrate was rejected, and a measured volume of the filtrate was diluted quantitatively with methanol to yield suitable concentrations lying in the linear range of the assay. 
I. A. Darwish et al.: High throughput microwell spectrophotometric assay for olmesartan medoxomil in tablets based on its charge-transfer reaction with DDQ, Acta Pharm. 64 (2014) 63-75.

\section{General analytical procedure}

Accurately measured aliquots $(100 \mu \mathrm{L})$ of the standard or sample solution containing varying amounts of OLM $(2-200 \mu \mathrm{g})$ were transferred into wells of 96-microwell assay plates. A hundred microliters of DDQ solution $\left(8.8 \times 10^{-2} \mathrm{~mol} \mathrm{~L}^{-1}\right)$ were added and the reaction was allowed to proceed at room temperature $\left(25 \pm 1{ }^{\circ} \mathrm{C}\right)$ for $5 \mathrm{~min}$. Absorbances of the resulting solutions were measured at $460 \mathrm{~nm}$ with the microplate reader. Blank wells were treated similarly except that $100 \mu \mathrm{L}$ of methanol was used instead of that sample, and absorbances of the blank wells were subtracted from those of the other wells.

\section{Determination of the molar ratio}

Job's method of continuous variation was employed. Master equimolar solutions $\left(2 \times 10^{-3} \mathrm{~mol} \mathrm{~L}^{-1}\right)$ of each of OLM and DDQ were prepared. Series of $200 \mu \mathrm{L}$ portions of the master solutions of OLM and DDQ were made up comprising different complementary ratios $(0: 10,1: 9, \ldots \ldots . . . . . .9: 1,10: 0$, inclusive) in each well of the 96-microwell assay plate. The reaction was allowed to proceed at room temperature (25 $\pm 1{ }^{\circ} \mathrm{C}$ ) for $5 \mathrm{~min}$. Absorbances were measured at $460 \mathrm{~nm}$ by the microwell-plate reader against blank wells. The measured absorbances were plotted as a function of the OLM mole fraction.

\section{Molecular modeling for the CT complex of OLM with DDQ}

Molecular modeling for the CT complex was performed using CS Chem3D Ultra, version 9 (Cambridge Soft Corporation, Cambridge, MA, USA) implemented with molecular orbital computations software (MOPAC) and molecular dynamics computations software (MM2).

\section{Validation of analytical procedures}

Linearity and sensitivity. - Under the optimal reaction conditions, the calibration curve for the analysis of OLM by the proposed assay was constructed by plotting absorbances as a function of the corresponding concentrations. The regression equation was derived using the least-squares method. The limits of detection $(L O D)$ and quantitation $(L O Q)$ were determined using the formula: $L O D$ or $L O Q=k \mathrm{SD}_{\mathrm{a}} / b$, where $k=3.3$ for $L O D$ and 10 for $L O Q, \mathrm{SD}_{\mathrm{a}}$ is the standard deviation of the intercept, and $b$ is the slope.

Accuracy and precision. - Accuracy of the proposed assay was assessed by analytical recovery studies. Recovery was determined by the standard addition method. Known amounts of OLM were added to pre-determined OLM-containing tablets (labeled to contain $20 \mathrm{mg}$ of OLM), and then determined by the proposed assay. The precision of the proposed assay was determined on samples of drug solutions at three concentration levels (5, 50 and $100 \mu \mathrm{g}$ per well). Five replicates of each concentration level were analyzed as a batch in a single assay run for evaluating the within-assay precision and on three consecutive days for evaluating the between-assay precision. 
Selectivity. - Interference from the congenital drugs that are co-formulated with OLM in some dosage forms was studied. The drugs were amlodipine besylate and hydrochlorothiazide. Potential interferences of these drugs were studied in a ratio normally present in their combined dosage forms, and recovery values were calculated.

Robustness and ruggedness. - Robustness was examined by evaluating the influence of small variations in assay variables on its analytical performance. In these experiments, one parameter was changed whereas the others were kept unchanged. Ruggedness was also tested by applying the proposed method to the OLM assay using the same operational conditions but using two different instruments (instrument 1 and 2) in two different laboratories and at different elapsed times. In each case, the percentage recovery was calculated. Instrument-1 was a microwell-plate absorbance reader (ELx 808, Bio-Tek Instruments Inc., USA) and instrument-2 was a microplate/cuvette reader (Spectramax M5, Molecular Devices, USA).

\section{RESULTS AND DISCUSSION}

\section{Assay development and design}

In this study, OLM was selected based on its therapeutic importance, clinical success, and the expected electron-donating ability. Previous studies involving CT reactions with polyhalo-/ polycyanoquinone electron $\pi$-acceptors revealed that DDQ is one of the most efficient reagents in terms of reactivity (19). Furthermore, its CT reaction with electron-donating analytes is instantaneous $(15,16)$. The 96-microwell design of the proposed assay was based on the previous success of Darwish et al. (20) in using this design for determination of some other pharmaceuticals.

\section{Reaction and spectral characteristics}

The interaction of OLM with DDQ was allowed to proceed at room temperature and the absorption spectrum of the produced chromogen was recorded. OLM gave red colored chromogen showing the absorption maximum at $460 \mathrm{~nm}$ (Fig. 2). This band was attributed to the formation of the radical anion $\mathrm{DDQ}^{-}(21)$, which was probably formed by the dissociation of an original donor-acceptor (D-A) complex:

\section{Optimization of experimental conditions}

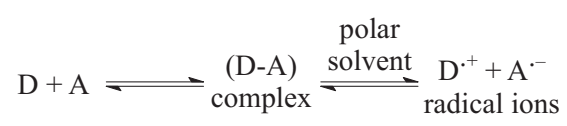

Optimization of experimental conditions affecting the reaction in the 96-well format was investigated by altering each reaction variable in turn while keeping the others constant. The OLM-DDQ complex exhibited the maximum absorption peak at $460 \mathrm{~nm}$. The results of variations in DDQ concentrations indicated that $4.4 \times 10^{-2} \mathrm{~mol} \mathrm{~L}^{-1}$ was the 
I. A. Darwish et al.: High throughput microwell spectrophotometric assay for olmesartan medoxomil in tablets based on its charge-transfer reaction with DDQ, Acta Pharm. 64 (2014) 63-75.

a)

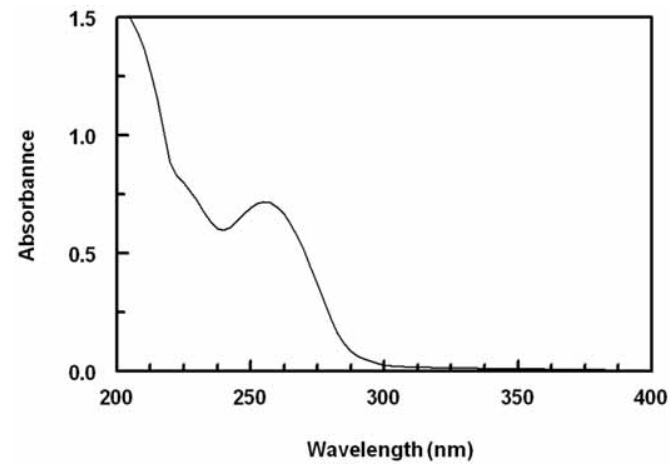

b)

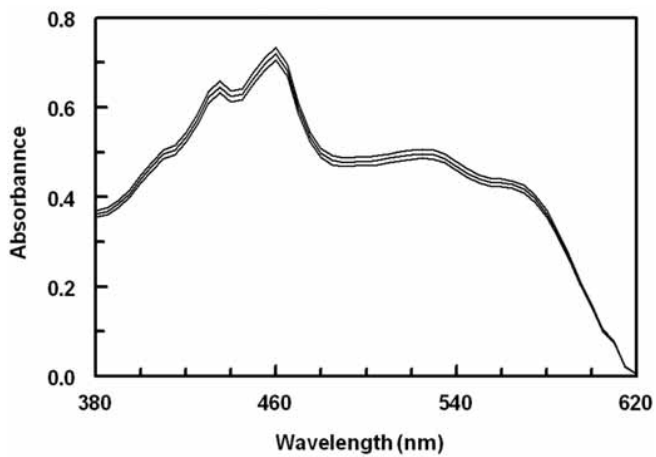

Fig. 2. Absorption spectra of: a) $20 \mu \mathrm{g} \mathrm{mL}-1$ of OLM; b) CT reaction product of DDQ with OLM alone (middle line) and OLM in the presence of amlodipine besylate (upper line) and hydrochlorothiazide (lower line).

optimum final DDQ concentration in the reaction mixture, since this concentration gave the highest absorbance. Previous studies (19) demonstrated that the interaction of electron donors with DDQ in polar solvents (e.g., methanol and acetonitrile) produced CT complexes with molar absorptivity values higher than those produced in non-polar sol-

Table I. Optimal conditions for the charge-transfer reaction of OLM with DDQ

\begin{tabular}{ccc}
\hline Condition & Studied range & Optimum \\
\hline DDQ conc. $\left(\mathrm{mol} \mathrm{L}^{-1}\right)$ & $0.44 \times 10^{-2}-17.6 \times 10^{-2}$ & $8.8 \times 10^{-2}$ \\
Solvent & Different ${ }^{\mathrm{a}}$ & Methanol \\
Reaction time $(\mathrm{min})$ & $0-30$ & 5 \\
Temperature $\left({ }^{\circ} \mathrm{C}\right)$ & $25-60$ & 25 \\
$\lambda_{\max }(\mathrm{nm})$ & $400-600$ & $460^{\mathrm{b}}$ \\
\hline
\end{tabular}


vents (e.g., chloroform). Different polar solvents were tested to prepare the DDQ solution: methanol, ethanol, 1-propanol, 1-butanol, and acetonitrile. Methanol offered the highest sensitivity; it was therefore selected. The optimum reaction time was determined by monitoring color development in the microwells at room temperature $\left(25 \pm 1{ }^{\circ} \mathrm{C}\right)$. Complete color development was attained instantaneously; however, for higher precision readings, the reaction was allowed to proceed for $5 \mathrm{~min}$. The developed color remained stable at room temperature for at least further $30 \mathrm{~min}$. A summary for the optimum conditions is given in Table I.

\section{Molar ratio, molecular modeling and site of interaction}

Job's method of continuous variation was used for determining the molar ratio of OLM to DDQ. It was concluded from the obtained Job's plot that the OLM/DDQ ratio was 1:1. This indicated that only one sites of interaction was involved in the formation of the colored CT complex in spite of the presence of more than one possible electron-donating sites in OLM structure (e.g., $\mathrm{OH}$ of the hydroxypropane-2-yl group and nitrogen atoms of the tetrazole ring). To investigate the site of interaction and postulate the reaction mechanism, modeling was performed for the CT complex. OLM and DDQ were energy-minimized alone and together. It was found that electron densities in the OLM molecule, located on the oxygen atom of $\mathrm{OH}$ of the hydroxypropane-2-yl group, the oxygen atom of the ester linkage and nitrogen atoms of the tetrazole were comparable

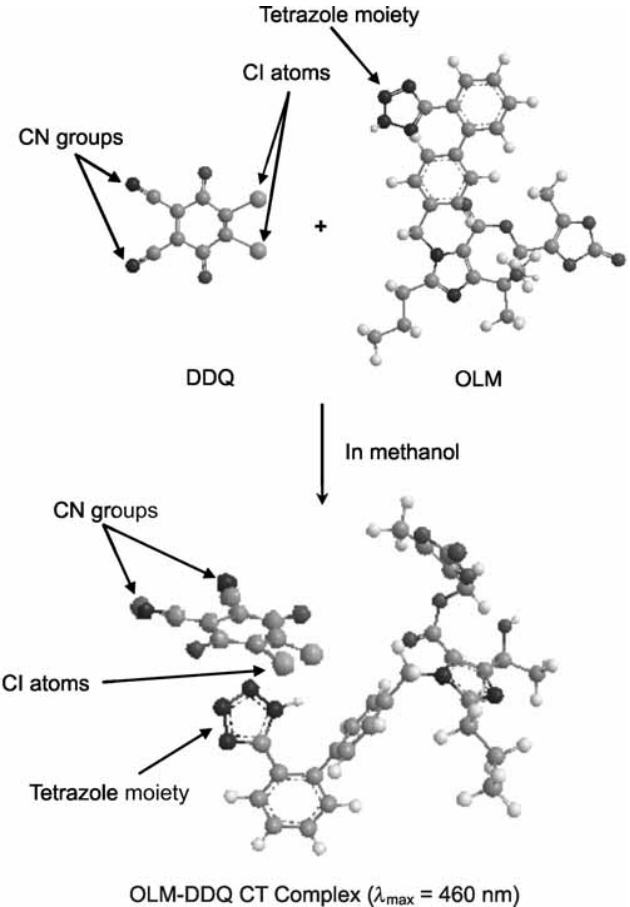

OLM-DDQ CT Complex $\left(\lambda_{\max }=460 \mathrm{~nm}\right)$
Fig. 3. 3D Model for the structures of DDQ, OLM and their energy-minimized CT complex in methanolic solution. 
I. A. Darwish et al.: High throughput microwell spectrophotometric assay for olmesartan medoxomil in tablets based on its charge-transfer reaction with DDQ, Acta Pharm. 64 (2014) 63-75.

$(-39428,-33456$, and -33085). Also, during the energy-minimization dynamics, it was found that the DDQ molecule moved toward the tetrazole moiety of OLM to form the CT complex (Fig. 3). These facts, taking the molar ratio in account, confirmed that only tetrazole was involved in the complex formation. The other anticipated centers are not likely to contribute to the $\mathrm{CT}$ reaction, probably due the steric hindrance effect of the OLM molecule (15).

\section{Validation of the proposed assay}

Linearity and sensitivity. - Beer law plot (10-points) was linear in the range of 2-200 $\mu \mathrm{g}$ per well with the correlation coefficient $R=0.9974$. The $L O D$ and $L O Q$ values were 0.53 and $1.61 \mu \mathrm{g}$ per well, respectively. Analytical parameters of the proposed assay are given in Table II.

Accuracy and precision. - Accuracy, expressed as recovery, was assessed and the mean analytical recovery was calculated. It was found to be $96.9-102.5 \%$ (Table III) indicating acceptable model accuracy of the proposed assay.

In assessing precision, the relative standard deviations (RSD) were 1.0-1.7 \% (Table II) proving good precision of the assay for routine application in quality control laboratories.

Table II. Parameters for the analysis of OLM by the proposed assay

\begin{tabular}{|c|c|}
\hline Parameter & Value \\
\hline Range ( $\mu$ g per well) & $2-200$ \\
\hline Intercept (absorbance unit) \pm RSD (\%) & $0.0171 \pm 16.06$ \\
\hline Slope \pm RSD $(\%)$ & $0.1122 \pm 4.99$ \\
\hline Correlation coefficient $(R)$ & 0.9974 \\
\hline$L O D$ ( $\mu$ g per well) & 0.53 \\
\hline$L O Q(\mu \mathrm{g}$ per well) & 1.61 \\
\hline \multicolumn{2}{|l|}{ Within-assay precision (RSD \%) ${ }^{\mathrm{a}}$} \\
\hline $5^{b}$ & 1.2 \\
\hline $50^{\mathrm{b}}$ & 1.0 \\
\hline $100^{\mathrm{b}}$ & 1.4 \\
\hline \multicolumn{2}{|l|}{ Between-assay precision (RSD \%) ${ }^{a}$} \\
\hline $5^{\mathrm{b}}$ & 1.5 \\
\hline $50^{\mathrm{b}}$ & 1.2 \\
\hline $100^{\mathrm{b}}$ & 1.7 \\
\hline
\end{tabular}

a $n=5$.

$\mathrm{b}$ Values are OLM concentrations in $\mu \mathrm{g}$ per well. 
I. A. Darwish et al.: High throughput microwell spectrophotometric assay for olmesartan medoxomil in tablets based on its charge-transfer reaction with DDQ, Acta Pharm. 64 (2014) 63-75.

Table III. Accuracy of the proposed 96-microwell-based spectrophotometric assay for OLM

\begin{tabular}{ccc}
\hline OLM added $(\mathrm{mg})$ & OLM found $(\mathrm{mg})$ & Recovery of OLM $(\% \pm \mathrm{SD})^{\mathrm{a}}$ \\
\hline 5.00 & 5.07 & $101.4 \pm 0.8$ \\
10.00 & 9.60 & $96.0 \pm 0.4$ \\
20.00 & 20.50 & $102.5 \pm 1.1$ \\
40.00 & 40.14 & $100.3 \pm 1.2$ \\
\hline
\end{tabular}

a Mean $\pm \mathrm{SD}, n=3$.

This high level of precision was, among others, attributed to the accuracy of the volumes that were concomitantly dispensed in the microwells by multi-channel pipettes, and completeness of the reaction in a small volume $(200 \mu \mathrm{L})$.

Selectivity. - The proposed assay has the advantage that the measurements are performed in the visible region, away from the UV-absorbing interfering substances that might be co-extracted from dosage forms containing OLM. Amlodipine besylate $(22,23)$ and hydrochlorothiazide $(24,25)$ are co-formulated with OLM in some dosage forms; their chemical structures are given in Fig. 1. Potential interferences of these drugs were studied and it was found that they exhibited no interferences with OLM in the proposed assay. This is evidenced from obtained good recovery values (97.9-99.8 \%, Table IV).

Selectivity of the CT reaction for OLM was attributed to its basic character (high electron densities found on nitrogen atoms of the tetrazole moeity), which allows formation of CT rather than hydrochlorothiazide, which does not have sufficient basicity to

Table IV. Selectivity study for reaction of OLM with DDQ in presence of co-formulated drugs

\begin{tabular}{|c|c|c|c|}
\hline \multicolumn{3}{|c|}{ Drug quantity (mg) } & \multirow{2}{*}{$\begin{array}{l}\text { Recovery of OLM } \\
(\% \pm S D)^{a}\end{array}$} \\
\hline OLM & Amlodipine besylate & Hydrochlorothiazide & \\
\hline 20.00 & 0.00 & 0.00 & $99.62 \pm 0.89$ \\
\hline 20.00 & 5.00 & 0.00 & $98.44 \pm 1.23$ \\
\hline 20.00 & 10.00 & 0.00 & $99.28 \pm 1.84$ \\
\hline 40.00 & 5.00 & 0.00 & $97.90 \pm 0.78$ \\
\hline 40.00 & 10.00 & 0.00 & $99.63 \pm 1.05$ \\
\hline 40.00 & 5.00 & 12.50 & $99.80 \pm 1.89$ \\
\hline 40.00 & 10.00 & 12.50 & $99.52 \pm 0.89$ \\
\hline 40.00 & 20.00 & 12.50 & $99.46 \pm 1.84$ \\
\hline
\end{tabular}

a Mean \pm SD, $n=3$. 
I. A. Darwish et al.: High throughput microwell spectrophotometric assay for olmesartan medoxomil in tablets based on its charge-transfer reaction with DDQ, Acta Pharm. 64 (2014) 63-75.

Table V. Robustness and ruggedness of the proposed 96-microwell-based spectrophotometric assay for OLM

\begin{tabular}{cc}
\hline Parameters & Recovery $(\% \pm \mathrm{SD})^{\mathrm{a}}$ \\
\hline & Robustness \\
\hline DDQ concentration $\left(\mathrm{mol} \mathrm{L}^{-1}\right)$ & \\
$8.6 \times 10^{-2}$ & $100.05 \pm 1.05$ \\
$9.0 \times 10^{-2}$ & \\
Reaction time (min) & $97.18 \pm 1.72$ \\
3 & $100.52 \pm 1.84$ \\
7 & \\
Temperature $\left({ }^{\circ} \mathrm{C}\right)$ & $97.89 \pm 1.82$ \\
23 & $100.54 \pm 1.91$ \\
28 & \\
\hline Instrument-to-instrument & \\
Instrument- 1 & $100.3 \pm 1.3$ \\
Instrument-2 & $98.4 \pm 1.0$ \\
Day-to-day & \\
Day-1 & \\
Day-2 & $100.2 \pm 1.1$ \\
Day-3 & $97.5 \pm 1.8$ \\
\hline
\end{tabular}

a Mean $\pm \mathrm{SD}, n=3$

achieve CT reaction. Although amlodipine has a basic character, it was co-formulated with OLM as besylate salt which did not show any ability for CT reaction with DDQ when tested by the proposed procedure under the proposed assay conditions. Recovery of OLM in the presence of amlodipine besylate was 98.2-100.1\%; however, it was 189.5 $-192.8 \%$ when amlodipine base was used. Further, no interference was observed from the excipients with the proposed assay, as indicated by good recovery values of 96.9-102.5\% (Table III). The absence of interference from the excipients, even though they contained basic component(s) was attributed to the extraction of OLM tablets prior to the analysis with methanol in which the excipients do not dissolve.

Robustness and ruggedness. - In evaluating the robustness, it was found that a small variation in one of the parameters did not significantly affect the procedures; recovery values were $97.2-100.5 \%$ (Table V). This indicated the reliability of the proposed assay during its routine application for the analysis of OLM. 
I. A. Darwish et al.: High throughput microwell spectrophotometric assay for olmesartan medoxomil in tablets based on its charge-transfer reaction with DDQ, Acta Pharm. 64 (2014) 63-75.

Table VI. Analysis of OLM in tablets by the reported and proposed methods

\begin{tabular}{ccccc}
\hline \multirow{2}{*}{ Formulation $^{\mathrm{a}}$} & \multicolumn{2}{c}{ Content of OLM $(\mathrm{mg} \text { per tablet })^{\mathrm{b}}$} & & \multirow{2}{*}{-value } \\
\cline { 2 - 3 } & Proposed method & Reported method $^{\mathrm{c}}$ & & $F_{\text {-value }}^{\mathrm{d}}$ \\
\hline Olmetec $^{\circledR}$ tablets & $20.56 \pm 0.29$ & $20.29 \pm 0.27$ & 1.57 & 1.12 \\
Azor $^{\circledR}$ tablets & $20.16 \pm 0.21$ & $19.88 \pm 0.14$ & 2.03 & 2.22 \\
Tribezor $^{\circledR}$ tablets & $19.88 \pm 0.15$ & $19.72 \pm 0.15$ & 1.29 & 1.08 \\
\hline
\end{tabular}

a All formulations contained $20 \mathrm{mg}$ of OLM; detailed composition were given in Experimental.

$\mathrm{b}$ Mean $\pm \mathrm{SD}, n=5$.

c The tabulated values at $95 \%$ confidence limit are 2.31 and 6.61 for $t$ - and for $F-$, respectively.

Ruggedness of the proposed method was evaluated as well and it was found that the variations lab-to-lab and day-to-day did not exceed $2 \%$ (Table V).

\section{Analysis of OLM tablets}

The commercially available OLM tablets (labeled to contain $20 \mathrm{mg}$ OLM) were subjected to analysis by the proposed and reported methods (11) the latter being based on the direct measurement of native UV absorption of OLM. The obtained results were then statistically compared. The exact content of OLM was 19.88-20.56 mg per tablet (Table VI). Values for $t$ and $F$ were calculated and found to be lower than the tabulated ones, indicating that there was no significant difference between, the proposed and the reported assays, at $95 \%$ confidence level, in terms of their accuracy and precision.

\section{CONCLUSIONS}

The present study describes the development and validation of a microwell spectrophotometric assay for the determination of OLM based on its CT reaction with DDQ reagent. The assay described herein offers the following advantages:

(i) Providing a high throughput analytical methodology that can facilitate the processing of a large number of samples in a relatively short time. This property was attributed to measuring the color signals in 96 wells in ca 30 seconds by the plate reader.

(ii) Reduction in the consumption of organic solvents, the exposure of the analyst to the toxic effects of organic solvent and reduction in the cost of analysis.

Acknowledgments. - The authors extend their appreciation to the Deanship of Scientific Research at King Saud University for the funding of this research through the research group project No. RGP-VPP-203. 


\section{REFERENCES}

1. M. Burnier and H. R. Brunner, Angiotensin II receptor antagonists, Lancet 355 (2000) 637-645; DOI: 10.1016/S0140-6736(99)10365-9.

2. G. K. Aulakh, R. K. Sodhi and M. Singh, An update on non-peptide angiotensin receptor antagonists and related RAAS modulators, Life Sci. 81 (2007) 615-639; DOI: 10.1016/j.lfs.2007.06.007.

3. P. D. Bari and A. R. Rote, RP-LC and HPTLC methods for the determination of olmesartan medoxomil and hydrochlorothiazide in combined tablet dosage forms, Chromatographia 69 (2009) 1469-1472; DOI: 10.1365/s10337-009-1094-z.

4. D. Liu, P. Hu, N. Matsushima, X. Li, L. Li and J. Jiang, Quantitative determination of olmesartan in human plasma and urine by liquid chromatography coupled to tandem mass spectrometry, J. Chromatogr. B. 856 (2007) 190-197; DOI: 10.1016/j.jchromb.2007.05.049.

5. V. V. Vaidya, M. N. Roy, S. M. Yetal, S. S. Joshi and S. A. Parekh, LC-MS-MS determination of olmesartan in human plasma. Chromatographia 67 (2008) 174-150; DOI: 10.1365/s10337-007-0453-x.

6. D. Liu, J. Jiang, P. Wang, S. Feng and P. Hu, Simultaneous quantitative determination of olmesartan and hydrochlorothiazide in human plasma and urine by liquid chromatography coupled to tandem mass spectrometry, J. Chromatogr. B 878 (2010) 743-748. DOI: 10.1016/j.jchromb.2010. 01.009 .

7. R. N. Sharma and S. S. Pancholi, RP-HPLC-DAD method for determination of olmesartan medoxomil in bulk and tablets exposed to forced conditions, Acta Pharm. 60 (2010) 13-24. DOI: 10.2478/v10007-010-0010-2.

8. N. J. Shah, B. N. Suhagia, R. R. Shah and N. M. Patel, Development and validation of a simultaneous HPTLC method for the estimation of olmesartan medoxomil and hydrochlorothiazide in tablet dosage form, Indian J. Pharm. Sci. 69 (2007) 834-836; DOI: 10.4103/0250-474X.58190.

9. C. K. Rao, K. K. Kumar, M. V. Srinivasulu, G. Madhusudhan, K. Mukkanti and K. S. Srinivas, Development and validation of stability-indicating LC method for olmesartan medoxamil, Am. J. Anal. Chem. 3 (2012) 153-160; DOI: 10.4236/ajac.2012.32022.

10. C. Mustafa and A. Sacide, Development of a CZE method for the determination of olmesartan medoxomil in tablets, Chromatographia 66 (2007) 929-933; DOI: 10.1365/s10337-007-0424-2.

11. M. Celebier and S. Altinoz, S. Determination of olmesartan medoxomil in tablets by uv-vis spectrophotometry, Pharmazie 62 (2007) 419-422; DOI: 10.1691/ph.2007.6.6233.

12. A. T. Hemke, M. V. Bhure, K. S. Chouhan, K. P. Gupta and S. G. Wadodkar, UV spectrophotometric determination of hydrochlorothiazide and olmesartan medoxomil in pharmaceutical formulation, E-J. Chem. 7 (2010) 1156-1161; DOI: 10.1155/2010/826585.

13. S. Caglar and A. Onal, Two simple and rapid spectrophotometric methods for the determination of a new antihypertensive drug olmesartan in tablets, J. Anal. Chem. 65 (2010) 239-243; DOI: 10.1134/S1061934810030056.

14. I. A. Darwish, Kinetic spectrophotometric assays for determination of trimetazidine dihydrochloride. Anal. Chim. Acta 551 (2005) 222-231; DOI: 10.1016/j.aca.2005.07.027.

15. I. A. Darwish, Analytical study for the charge transfer complexes of losartan potassium, Anal. Chim. Acta 549 (2005) 212-220; DOI: 10.1016/j.aca.2005.06.023.

16. I. A. Darwish, H. A. Abdel-Wadood and N. A. Abdel-Latif, Validated spectrophotometric and fluorimetric assays for analysis of clozapine in tablets and urine, Ann. Chim. 95 (2005) 345-356; DOI: 10.1002 /adic.200590039.

17. A. T. Fidler, E. L. Baker and R. E. Letz, Neurobehavioural effects of occupational exposure to organic solvents among construction painters, Br. J. Ind. Med. 44 (1987) 292-308; DOI: 10.1136/ oem.44.5.292. 
I. A. Darwish et al.: High throughput microwell spectrophotometric assay for olmesartan medoxomil in tablets based on its charge-transfer reaction with DDQ, Acta Pharm. 64 (2014) 63-75.

18. H. Wennborg, J. P. Bonde, M. Stenbeck and J. Olsen, Adverse reproduction outcomes among employees working in biomedical research laboratories, Scand. J. Work Environ. Health 28 (2002) 5-11; DOI: 10.5271/sjweh.640.

19. H. F. Askal, Spectrophotometric study of the charge transfer complexes of some pharmaceutical butyrophenones, Talanta 44 (1997) 1749-1755; DOI: 10.1016/S0039-9140(97)00043-X.

20. I. A. Darwish, A. M. Mahmoud and A. A. Al-Majed, A novel analytical approach for reducing the consumption of organic solvents in the charge transfer-based spectrophotometric analysis: application in the analysis of certain antihypertensive drugs, Acta Pharm. 60 (2010) 493-501; DOI: $10.2478 / v 10007-010-0035-6$.

21. A. Taha and G. Rücker, Utility of pi-acceptors in alkaloid assay, Arch. Pharm. (Weinheim) 310 (1977) 485-494; DOI: 10.1002/ardp.19773100608.

22. C. Rosendorff, R. Dubiel, J. Xu and K. J. Chavanu, Comparison of olmesartan medoxomil versus amlodipine besylate on regression of ventricular and vascular hypertrophy, Am. J. Cardiol. 104 (2009) 359-365; DOI: 10.1016/j.amjcard.2009.03.042.

23. S. G. Chrysant, Amlodipine besylate/olmesartan medoximil fixed combination for the treatment of hypertension, Expert Rev. Cardiovasc. Ther. 8 (2009) 887-895; DOI: 10.1586/erc.09.85.

24. D. J. Kereiakes, J. M. Neutel, H. A. Punzi, J. Xu, L. J. Lipka and R. Dubiel, Efficacy and safety of olmesartan medoxomil and hydrochlorothiazide compared with benazepril and amlodipine besylate, Am. J. Cardiovasc. Drugs 7 (2007) 361-372; DOI: 10.2165/00129784-200707050-00006.

25. S. G. Chrysant, M. A. Weber, A. C. Wang and D. J. Hinman, Evaluation of antihypertensive therapy with the combination of olmesartan medoxomil and hydrochlorothiazide, Am. J. Hypertens. 17 (2004) 252-259; DOI: 10.1016/j.amjhyper.2003.11.003. 